\title{
Neutral Pentacoordinate Silicon Fluorides Derived from Amidinate, Guanidinate, and Triazapentadienate Ligands and Base-Induced Disproportionation of $\mathrm{Si}_{2} \mathrm{Cl}_{6}$ to Stable Silylenes
}

\author{
Rajendra S. Ghadwal, ${ }^{\dagger}$ Kevin Pröpper, ${ }^{\dagger}$ Birger Dittrich, ${ }^{\dagger}$ Peter G. Jones, ${ }^{\ddagger}$ and Herbert W. Roesky ${ }^{*, \dagger}$ \\ ${ }^{\dagger}$ Institut für Anorganische Chemie der Universität Göttingen, Tammannstrasse 4, D 37077 Göttingen, Germany, \\ and Institut für Anorganische und Analytische Chemie der Technischen Universität Braunschweig, \\ Hagenring 30, D 38106 Braunschweig, Germany
}

Received October 11, 2010

\begin{abstract}
Pentacoordinate silicon fluorides $\mathrm{L}^{1} \mathrm{SiF}_{3}(\mathbf{2 a}), \mathrm{L}^{2} \mathrm{SiF}(\mathbf{2 b})$, and $\left(\mathrm{L}^{3} \mathrm{SiF}\right)_{2}(\mathbf{2} \mathbf{c})_{2}$ based on amidinate $\left(\mathrm{L}^{1}=\mathrm{PhC}\left(\mathrm{NB}^{t} \mathrm{Bu}\right)_{2}\right)$, guanidinate $\left(\mathrm{L}^{2}=1,3,4,6,7,8\right.$-hexahydro-2 $\mathrm{H}$-pyrimido[1,2-a]pyrimidinate), and triazapentadienate $\left(\mathrm{L}^{3}=\mathrm{NC}\left(\mathrm{NMe}_{2}\right) \mathrm{NC}\right.$ $\left.\left(\mathrm{NMe}_{2}\right) \mathrm{NAr} ; \mathrm{Ar}=2,6-{ }^{i} \mathrm{Pr}_{2} \mathrm{C}_{6} \mathrm{H}_{3}\right)$ ligands were prepared by fluorination of the corresponding chlorosilanes $\mathrm{L}^{1} \mathrm{SiCl}_{3}(1 \mathrm{a})$, $\mathrm{L}^{2} \mathrm{SiCl}_{3}(\mathbf{1 b})$, and $\mathrm{L}^{3} \mathrm{SiCl}_{2}$ (1c) with $\mathrm{Me}_{3} \mathrm{SnF}$ at ambient temperature. Compounds $1 \mathbf{b}, 1 \mathbf{c}, \mathbf{2 a}, \mathbf{2 b}$, and $(\mathbf{2 c})_{2}$ were characterized by ${ }^{1} \mathrm{H},{ }^{13} \mathrm{C},{ }^{19} \mathrm{~F}$, and ${ }^{29} \mathrm{Si} N M R$ spectroscopic studies. Molecular structures of $1 \mathbf{b}, 1 \mathbf{c}, 2 \mathrm{a}$, and $(\mathbf{2 c})_{2}$ were determined by single crystal $\mathrm{X}$-ray structural analysis. Invariom refinement involving non-spherical scattering factors of the Hansen-Coppens multipole model was performed for $\mathbf{1 b}$. Compound $\mathrm{L}^{3} \mathrm{SiF}_{2}(\mathbf{2 c})$ is dimeric both in the solid state and in solution, whereas its chloro-analogue $1 \mathrm{c}$ is monomeric. The attempted synthesis of diamidinatotetrachlorodisilane by reaction of lithium amidinate with $\mathrm{Si}_{2} \mathrm{Cl}_{6}$ led to the formation of the silane (1a) and the silylene $\mathrm{L}^{1} \mathrm{SiCl}(3)$. Reaction of $\mathrm{Si}_{2} \mathrm{Cl}_{6}$ with $\mathrm{N}$-heterocyclic carbenes (NHC) afforded $\mathrm{NHC}$ adducts of dichlorosilylene and $\mathrm{SiCl}_{4}$. A one pot method for the preparation of base-stabilized silylenes from $\mathrm{Si}_{2} \mathrm{Cl}_{6}$ is discussed.
\end{abstract}

\section{Introduction}

Compounds with coordination numbers greater than four at the silicon have received considerable attention during the past few decades. Silicon(IV) halides are well-known as Lewis acids, and compounds with hypervalent silicon were first observed at the beginning of the 19 th century. ${ }^{1}$ Compounds with higher-coordinate silicon have been of great interest both for experimental ${ }^{2,3}$ and theoretical ${ }^{4}$ scientists because of their unusual bonding properties, ${ }^{5}$ distinct reactivity, and potential as synthons ${ }^{6}$ for preparing novel silicon compounds. Moreover, compounds with higher-coordinate silicon are known to act as reactive intermediates ${ }^{7}$ and as reagents for preparing organosilicon compounds. ${ }^{8}$ On the basis of the size of the central atoms (carbon and silicon), the enhanced stability of compounds with pentacoordinate silicon has been theoretically ${ }^{4 \mathrm{~g}}$ explained. Ionic compounds ${ }^{3}$ with higher-coordinate silicon are well documented, but similar compounds with neutral ligands are scarce. ${ }^{2}$

Moreover, a recent growth area in main group chemistry has been the isolation of compounds with elements in unusual oxidation states. ${ }^{9,10}$ The synthesis, characterization, and applications of compounds with low valent main group elements stabilized kinetically by bulky ligands and/or thermodynamically

*To whom correspondence should be addressed. E-mail: hroesky@gwdg.de. (1) Davy, J. Phil. Trans. R. Soc. London 1812, 102, 352-369. by donor atoms $(\mathrm{C}, \mathrm{N}$, or $\mathrm{P})$ have proved to be attractive topics of research. Further progress in this field is driven by the design and synthesis of new compounds with main group elements.

(2) (a) Frye, C. L.; Vogel, G. E.; Hall, J. A. J. Am. Chem. Soc. 1961, 83, 996 997. (b) Onan, K. D.; McPhail, A. T.; Yoder, C. H.; Hillyard, R. W. J. Chem. Soc., Chem. Commun. 1978, 209-210. (c) Voronkov, M. G.; Frolov, Yu. L.; D'yakov, V. M.; Chipanina, N. N.; Gubanova, L. I.; Gavrilova, G. A.; Klyba, L. V.; Aksamentova, T. N. J. Organomet. Chem. 1980, 201, 165-177. (d) Corriu, R. J. P.; Royo, G.; De Saxcé, A. J. Chem. Soc., Chem. Commun. 1980, 892-894. (e) Klebe, G.; Hensen, K.; Fuess, H. Chem. Ber. 1983, 116, 3125-3132. (f) Farnham, W. B.; Whitney, J. F. J. Am. Chem. Soc. 1984, 106, 3992-3994. (g) Corriu, R. J. P.; Mazhar, M.; Poirier, M.; Royo, G. J. Organomet. Chem. 1986, 306, C5-C9. (h) Macharashvili, A. A.; Shklover, V. E.; Struchkov, Yu. T.; Oleneva, G. I.; Kramarova, E. P.; Shipov, A. G.; Baukov, Yu. I. J. Chem. Soc., Chem. Commun. 1988, 683-685. (i) Boyer, J.; Brelière, C.; Carré, F.; Corriu, R. J. P.; Kpoton, A.; Poirier, M.; Royo, G.; Young, J. C. J. Chem. Soc., Dalton Trans. 1989, 43-51. (j) Gudat, D.; Verkade, J. G. Organometallics 1989, 8, 2772-2779. (k) Kobayashi, J.; Kawaguchi, K.; Kawashima, T. J. Am. Chem. Soc. 2004, 126, 16318-16319. (1) Pongor, G.; Kolos, Z.; Szalay, R.; Knausz, D. J. Mol. Struct. (THEOCHEM) 2005, 714, 87-97. (m) Szalay, R.; Pongor, G.; Harmat, V.; Böcskei, Z.; Knausz, D. J. Organomet. Chem. 2005, 690, 1498-1506. (n) Kalikhman, I.; Gostevskii, B.; Kertsnus, E.; Deuerlein, S.; Stalke, D.; Botoshansky, M.; Kost, D. J. Phys. Org. Chem. 2008, 21, 1029-1034. (o) Metz, S.; Burschka, C.; Tacke, R. Organometallics 2008, 27, 6032-6034. (p) Metz, S.; Burschka, C.; Tacke, R. Organometallics 2009, 28, 2311-2317. (q) Fester, G. W.; Wagler, J.; Brendler, E.; Böhme, U.; Gerlach, D.; Kroke, E. J. Am. Chem. Soc. 2009, 131, 6855-6864. (r) Kalikhman, I.; Kertsnus-Banchik, E.; Gostevskii, B.; Kocher, N.; Stalke, D.; Kost, D. Organometallics 2009, 28, 512-516. (s) Ghadwal, R. S.; Sen, S. S.; Roesky, H. W.; Tavcar, G.; Merkel, S.; Stalke, D. Organometallics 2009, 28, 6374-6377. 
Herein we report on the synthesis and characterization of pentacoordinate silicon fluorides containing bidentate mono(amidinate, guanidinate) and bifunctional (triazapentadienate) ligands by efficient fluorination of their chloro analogues 1a-1c with $\mathrm{Me}_{3} \mathrm{SnF}$. The synthesis and characterization of $\mathrm{L}^{2} \mathrm{SiCl}_{3}(\mathbf{1 b})$ and $\mathrm{L}^{3} \mathrm{SiCl}_{2}$ (1c) are reported for the first time.

One pot methods for the synthesis of compounds with low valent elements are undoubtedly important to obtain sufficient amount of starting material for new reactions. Recently we reported ${ }^{6 \mathrm{~d}}$ a nearly quantitative $(79 \%$ yield) synthesis of NHC-stabilized dichlorosilylenes by a clean one-step reaction of $\mathrm{NHC}$ with $\mathrm{HSiCl}_{3}$. We further described ${ }^{6 f}$ the highyield synthesis of monochlorosilylene $\mathrm{L}^{1} \mathrm{SiCl}$ from $\mathrm{L}^{1} \mathrm{SiHCl}_{2}$

(3) (a) Bassindale, A. R.; Taylor, P. G. In The Chemistry of Organic Silicon Compounds; Patai, S., Rappoport, Z., Eds.; John Wiley \& Sons Ltd.: New York, 1989; Part 1, pp 839-892. (b) Corriu, R. J. P.; Young, J. C. In The Chemistry of Organic Silicon Compounds; Patai, S., Rappoport, Z., Eds.; John Wiley \& Sons: Chichester, U.K., 1989; Part 2, pp 1241-1288. (c) Johnson, S. E.; Day, R. O.; Holmes, R. R. Inorg. Chem. 1989, 28, 3182-3189. (d) Holmes, R. R. Chem. Rev. 1990, 90, 17-31. (e) Kost, D.; Gostevskii, B.; Kalikhman, I. Pure Appl. Chem. 2007, 79, 1125-1134. (f) Prince, P. D.; Bearpark, M. J.; McGrady, G. S.; Steed, J. W. J. Chem. Soc., Dalton Trans. 2008, 271-282. (g) Kost, D.; Kalikhman, I. Acc. Chem. Res. 2009, 42, 303-314.

(4) (a) Marsden, C. J. Inorg. Chem. 1983, 22, 3177-3178. (b) Hu, J.; Schaad, L. J.; Hess, B. A., Jr. J. Am. Chem. Soc. 1991, 113, 1463-1464. (c) Alkorta, I.; Rozas, I.; Elguero, J. J. Phys. Chem. A 2001, 105, 743-749. (d) Ignatyev, I. S.; Schaefer, H. F., III. J. Phys. Chem. A 2001, 105, 7665-7671. (e) Fleischer, H. Eur. J. Inorg. Chem. 2001, 393-404. (f) Davydova, E. I.; Timoshkin, A. Y.; Sevastianova, T. N.; Suvorov, A. V.; Frenking, G. J. Mol. Struct. THEOCHEM 2006, 767, 103-111. (g) Pierrefixe, S. C. A. H.; Guerra, C. F.; Bickelhaupt, F. M. Chem.-Eur. J. 2008, 14, 819-828. (h) Couzijn, E. P. A.; van den Engel, D. W. F.; Slootweg, J. C.; de Kanter, F. J. J.; Ehlers, A. W.; Schakel, M.; Lammertsma, K. J. Am. Chem. Soc. 2009, 131, 3741-3751. (i) Hallóczki, O.; Nyulászi, L. Organometallics 2009, 28, 4159-4164

(5) Kocher, N.; Henn, J.; Gostevskii, B.; Kost, D.; Kalikhman, I.; Engels, B.; Stalke, D. J. Am. Chem. Soc. 2004, 126, 5563-5568.

(6) (a) So, C.-W.; Roesky, H. W.; Magull, J.; Oswald, R. B. Angew. Chem., Int. Ed. 2006, 45, 3948-3950. So, C.-W.; Roesky, H. W.; Magull, J.; Oswald, R. B. Angew. Chem. 2006, 118, 4052-4054. (b) So, C.-W.; Roesky, H. W.; Gurubasavaraj, P. M.; Oswald, R. B.; Gamer, M. T.; Jones, P. G.; Blaurock, S. J. Am. Chem. Soc. 2007, 129, 12049-12054. (c) Wang, Y.; Xie, Y.; Wei, P.; King, R. B.; Schaefer, H. F., III; Schleyer, P. v. R.; Robinson, G. H. Science 2008, 321, 1069-1071. (d) Ghadwal, R. S.; Roesky, H. W.; Merkel, S.; Henn, J.; Stalke, D. Angew. Chem. Int. Ed. 2009, 48, 5683-5686. Ghadwal, R. S.; Roesky, H. W. Merkel, S.; Henn, J.; Stalke, D. Angew. Chem. 2009, 121, 5793-5796. (e) Ghadwal, R. S.; Sen, S. S.; Roesky, H. W.; Granitzka, M.; Kratzert, D.; Merkel, S.; Stalke, D. Angew. Chem., Int. Ed. 2010, 49, 3952-3955. Angew. Chem. 2010, 122, 4044-4047. (f) Sen, S. S.; Roesky, H. W.; Stern, D.; Henn, J.; Stalke, D. J. Am. Chem. Soc. 2010, 132, 1123-1126.

(7) (a) Tandura, S. N.; Voronkov, M. G.; Alekseev, N. V. Top. Curr. Chem. 1986, 131, 99-189. (b) Chuit, C.; Corriu, R. J. P.; Reye, C.; Young, J. C. Chem. Rev. 1993, 93, 1371-1448. (c) Chuit, C.; Corriu, R. J. P.; Reye, C. In Chemistry of Hypervalent Compounds; Akiba, K., Ed.; Wiley-VCH: New York, 1999, pp 81-146. (d) Kira, M.; Zhang, L. C. In Chemistry of Hypervalent Compounds; Akiba, K., Ed.; Wiley-VCH: New York, 1999, pp 147-170. (e) Katsukiyo, M.; Hosomi, A. In Main Group Metals in Organic Synthesis; Yamamoto, H., Oshima, K., Eds.; Wiley-VCH: Weinheim, 2004; Vol. 2, 409-592. (f) Rendler, S.; Oestreich, M. Synthesis 2005, 1727-1747. (g) Denmark, S. E. J. Org. Chem. 2009, 74, 2915-2927.

(8) (a) Denmark, S. E.; Wynn, T.; Beutner, G. L. J. Am. Chem. Soc. 2002, 124, 13405-13407. (b) Denmark, S. E.; Beutner, G. L.; Wynn, T.; Eastgate, M. D. J. Am. Chem. Soc. 2005, 127, 3774-3789. (c) Denmark, S. E.; Fujimori, S. In Modern Aldol Reactions; Mahrwald, R., Ed.; Wiley-VCH: Weinheim, 2004, Vol. 2, Chapter 7, pp 229-326.

(9) (a) Power, P. P. Chem. Rev. 1999, 99, 3463-3503. (b) Power, P. P. In Struct. Bonding (Berlin); Roesky, H. W., Atwood, D. A., Eds.; Springer-Verlag: Berlin, 2002; Vol. 103, pp 57-84. (c) Fischer, R. C.; Power, P. P. Chem. Rev. 2010, 110, 3877-3923. (c) Mizuhata, Y.; Sasamori, T.; Tokitoh, N. Chem. Rev. 2009, 109, 3479-3511

(10) (a) Jones, C. Coord. Chem. Rev. 2010, 254, 1273-1289. (b) Power, P. P. Nature 2010, 463, 171-177. (c) Haaf, M.; Schmedake, T. A.; West, R. Acc. Chem. Res. 2000, 33, 704-714. (d) Mandal, S. K.; Roesky, H. W. Chem. Commun. 2010, 46, 6016-6041. using $\mathrm{NHC}$ or $\mathrm{LiN}\left(\mathrm{SiMe}_{3}\right)_{2}$ as a dehydrochlorinating agent. As a continuation of these studies, we report herein a new method for the preparation of base-stabilized silylenes from $\mathrm{Si}_{2} \mathrm{Cl}_{6}$.

\section{Experimental Section}

General Procedures. All manipulations were carried out under an inert atmosphere of nitrogen using standard Schlenk line techniques or a glovebox. The solvents used were purified by MBRAUN solvent purification system MB SPS-800. Compound $1 \mathbf{c}^{6 \mathrm{a}}$ and $\mathrm{L}^{3^{\prime}} \mathrm{Li}\left(\mathrm{L}^{3^{\prime}}=\mathrm{Me}_{3} \mathrm{SiNC}\left(\mathrm{NMe}_{2}\right) \mathrm{NC}\left(\mathrm{NMe}_{2}\right) \mathrm{NAr}\right.$ and $\mathrm{Ar}$ is 2,6- $\left.{ }^{i} \mathrm{Pr}_{2} \mathrm{C}_{6} \mathrm{H}_{3}\right)^{11}$ were prepared according to literature methods. All chemicals received from Aldrich were used without further purification. $\mathrm{C}_{6} \mathrm{D}_{6}$ and $\mathrm{THF}-\mathrm{d}_{8}$ were dried over $\mathrm{Na}$ metal and distilled under nitrogen prior to use. ${ }^{1} \mathrm{H},{ }^{13} \mathrm{C},{ }^{19} \mathrm{~F}$, and ${ }^{29} \mathrm{Si} \mathrm{NMR}$ spectra were recorded using Bruker Avance DPX 200 or Bruker Avance DRX 500 spectrometers. Elemental analyses were obtained from the Analytical Laboratory of the Institute of Inorganic Chemistry at the University of Göttingen.

Preparation of $\mathrm{L}^{2} \mathrm{SiCl}_{3}$ (1b). To $100 \mathrm{~mL}$ of a diethyl ether solution of $1,3,4,6,7,8$-hexahydro- $2 H$-pyrimido[1,2-a]pyrimidine $\left(\mathrm{L}^{2} \mathrm{H}\right)(1.54 \mathrm{~g}, 11.06 \mathrm{mmol})$ was added a $2.5 \mathrm{M}$ solution of ${ }^{t} \mathrm{BuLi}$ (4.45 mL, $11.12 \mathrm{mmol})$ at $-78^{\circ} \mathrm{C}$, and the mixture was stirred for $4 \mathrm{~h}$ at room temperature. Into the resulting white suspension $\mathrm{SiCl}_{4}(1.30 \mathrm{~mL}, 11.34 \mathrm{mmol})$ was syringed at $-78^{\circ} \mathrm{C}$ with constant stirring. The reaction mixture was further stirred overnight. The volatiles were removed under vacuum to obtain a white solid. The solid was dissolved in toluene $(50 \mathrm{~mL})$ and filtered through Celite. The resulting filtrate was reduced to $25 \mathrm{~mL}$ under vacuum and stored at $-35^{\circ} \mathrm{C}$ in a freezer to yield compound $\mathbf{1 b}$ as colorless crystals $(2.71 \mathrm{~g}, 90 \%)$. Anal. Calcd $(\%)$ for $\mathrm{C}_{7} \mathrm{H}_{12} \mathrm{Cl}_{3} \mathrm{~N}_{3} \mathrm{Si}(\mathrm{M}=$ 272.63): C, 30.84; H, 4.44; N, 15.41. Found (\%): C, 30.79; H, 4.34; $\mathrm{N}, 15.29 .{ }^{1} \mathrm{H} \mathrm{NMR}\left(200 \mathrm{MHz}, \mathrm{C}_{6} \mathrm{D}_{6}, 298 \mathrm{~K}\right): \delta 1.16\left(\mathrm{~m}, 4 \mathrm{H}, \mathrm{CH}_{2}\right)$, $2.11(\mathrm{t}, J=5.6 \mathrm{~Hz}, 4 \mathrm{H}, \mathrm{CH}), 2.93\left(\mathrm{t}, J=5.6 \mathrm{~Hz}, 4 \mathrm{H}, \mathrm{CH}_{2}\right) \mathrm{ppm}$. ${ }^{13} \mathrm{C}\left\{{ }^{1} \mathrm{H}\right\}$ NMR $\left(75 \mathrm{MHz}, \mathrm{C}_{6} \mathrm{D}_{6}, 298 \mathrm{~K}\right): \delta 22.53\left(\mathrm{CH}_{2}\right), 37.54$ $\left(\mathrm{CH}_{2}\right), 44.04\left(\mathrm{CH}_{2}\right), 154.62\left(\mathrm{CN}_{3}\right)$ ppm. ${ }^{29} \mathrm{Si} \mathrm{NMR}(59 \mathrm{MHz}$, $\left.\mathrm{C}_{6} \mathrm{D}_{6}, 298 \mathrm{~K}\right): \delta-103.42 \mathrm{ppm}$.

$\mathbf{L}^{3} \mathrm{SiCl}_{\mathbf{2}}$ (1c). $\mathrm{SiCl}_{4}(0.82 \mathrm{~mL}, 7.12 \mathrm{mmol})$ was added to $100 \mathrm{~mL}$ of a diethyl ether solution of $\mathrm{L}^{3^{\prime}} \mathrm{Li}(2.82 \mathrm{~g}, 7.12 \mathrm{mmol})$ at $-78^{\circ} \mathrm{C}$, and then the reaction mixture was allowed to warm to room temperature. After overnight stirring the $\mathrm{LiCl}$ formed was filtered off. Removal of all volatiles from the filtrate resulted in a white solid. Recrystallization from toluene $(20 \mathrm{~mL})$ afforded compound $\mathbf{1 c}$ as colorless crystals $(2.14 \mathrm{~g}, 72 \%)$. Anal. Calcd. for $\mathrm{C}_{18} \mathrm{H}_{29} \mathrm{Cl}_{2} \mathrm{~N}_{5} \mathrm{Si}$ $(\mathrm{M}=414.45): \mathrm{C}, 52.16 ; \mathrm{H}, 7.05 ; \mathrm{N}, 16.90$. Found (\%): C, 52.19; H, 7.08; N, 16.83. ${ }^{1} \mathrm{H}$ NMR $\left(200 \mathrm{MHz}, \mathrm{C}_{6} \mathrm{D}_{6}, 298 \mathrm{~K}\right): \delta 1.06(\mathrm{~d}, J=$ $6.7 \mathrm{~Hz}, 6 \mathrm{H}, \mathrm{CHMe}$ ), 1.30 (d, $J=6.7 \mathrm{~Hz}, 6 \mathrm{H}, \mathrm{CHMe}$ ), 2.24 (s, 6H, NMe $\left.e_{2}\right), 2.94$ (s, 3H, NMe 2 ), 2.97 (s, 3H, NMe $), 3.27$ (m, $2 \mathrm{H}, \mathrm{CHMe}$ ) $6.98-7.05\left(\mathrm{~m}, 3 \mathrm{H}, \mathrm{C}_{6} \mathrm{H}_{3}\right) \mathrm{ppm} .{ }^{13} \mathrm{C}\left\{{ }^{1} \mathrm{H}\right\} \mathrm{NMR}$ $\left(75 \mathrm{MHz}, \mathrm{C}_{6} \mathrm{D}_{6}, 298 \mathrm{~K}\right): \delta 23.61(\mathrm{CHMe}), 25.78\left(\mathrm{CHMe}_{2}\right), 28.46$ $\left(\mathrm{CHMe}_{2}\right), 37.01,37.07,39.50\left(\mathrm{~N} M e_{2}\right), 125.10,127.96,137.42$ $\left(C_{6} \mathrm{H}_{3}\right), 146.57$ (ipso- $\left.C_{6} \mathrm{H}_{3}\right), 161.66,162.31(\mathrm{NCN})$ ppm. ${ }^{29} \mathrm{Si}$ NMR (59 MHz, $\left.\mathrm{C}_{6} \mathrm{D}_{6}, 298 \mathrm{~K}\right): \delta-30.57 \mathrm{ppm}$.

$\mathbf{L}^{\mathbf{1}} \mathbf{S i F}_{\mathbf{3}}$ (2a). THF $(50 \mathrm{~mL})$ was added to $\mathbf{1 a}(1.75 \mathrm{~g}, 4.87 \mathrm{mmol})$ and $\mathrm{Me}_{3} \mathrm{SnF}$ (2.68 g, $16.66 \mathrm{mmol}$ ) in a Schlenk flask at room temperature. Stirring of the reaction mixture for $20 \mathrm{~min}$ resulted in the dissolution of $\mathrm{Me}_{3} \mathrm{SnF}$ and the formation of a colorless solution. After further stirring for $2 \mathrm{~h}$, all volatiles were removed under vacuum to obtain a white solid. Recrystallization from toluene $(20 \mathrm{~mL})$ at $-35^{\circ} \mathrm{C}$ afforded $\mathbf{2 a}(1.13 \mathrm{~g}, 75 \%)$ as colorless crystals after $20 \mathrm{~h}$. Anal. Calcd ( $\%)$ for $\mathrm{C}_{15} \mathrm{H}_{23} \mathrm{~F}_{3} \mathrm{~N}_{2} \mathrm{Si}(\mathrm{M}=316.44)$ : $\mathrm{C}$, 56.93; H, 7.33; N, 8.85. Found (\%): C, 56.79; H, 7.30; N, 8.72. ${ }^{1} \mathrm{H}$ NMR (200 MHz, $\left.\mathrm{C}_{6} \mathrm{D}_{6}, 298 \mathrm{~K}\right): \delta 1.05$ (s, 18H, CMe $), 6.70-6.88$ $\left(\mathrm{m}, 5 \mathrm{H}, \mathrm{C}_{6} H_{5}\right) \mathrm{ppm} .{ }^{13} \mathrm{C}\left\{{ }^{1} \mathrm{H}\right\} \mathrm{NMR}\left(125 \mathrm{MHz}, \mathrm{THF}-d_{8}, 298 \mathrm{~K}\right): \delta$ $31.44\left(\mathrm{CMe}_{3}\right), 54.52\left(\mathrm{CMe}_{3}\right), 128.31,128.97,129.11,129.20$,

(11) Zhou, M.; Song, Y.; Gong, T.; Tong, H.; Guo, J.; Weng, L.; Liu, D. Inorg. Chem. 2008, 47, 6692-6700. 
Table 1. Crystallographic Data and Structure Refinement for $\mathbf{1 b}$ and $\mathbf{1 c}$

\begin{tabular}{|c|c|c|}
\hline & $1 b$ & 1c \\
\hline formula & $\mathrm{C}_{7} \mathrm{H}_{12} \mathrm{Cl}_{3} \mathrm{~N}_{3} \mathrm{Si}$ & $\mathrm{C}_{18} \mathrm{H}_{29} \mathrm{Cl}_{2} \mathrm{~N}_{5} \mathrm{Si}$ \\
\hline $\mathrm{F}_{W}$ & 272.64 & 414.45 \\
\hline cryst size $/ \mathrm{mm}$ & $0.50 \times 0.20 \times 0.15$ & $0.40 \times 0.40 \times 0.25$ \\
\hline cryst syst & monoclinic & triclinic \\
\hline space group & $P 2_{1} / c$ & $P \overline{1}$ \\
\hline$T /{ }^{\circ} \mathrm{C}$ & -173 & -173 \\
\hline$a / \AA$ & $10.6177(3)$ & $10.1387(4)$ \\
\hline$b / \AA$ & $8.1230(2)$ & $14.3972(6)$ \\
\hline$c / \AA$ & $13.5089(4)$ & $15.9813(6)$ \\
\hline$\alpha / \operatorname{deg}$ & 90 & $71.159(4)$ \\
\hline$\beta / \mathrm{deg}$ & $101.155(1)$ & $75.077(4)$ \\
\hline$\gamma / \operatorname{deg}$ & 90 & $85.845(4)$ \\
\hline$V / \AA^{3}$ & $1143.10(5)$ & $2133.18(15)$ \\
\hline$D_{\text {calcd }} / \mathrm{g} \mathrm{cm}^{-3}$ & 1.584 & 1.290 \\
\hline$Z$ & 4 & 4 \\
\hline wavelength/ ̊ & 1.54184 & 0.71073 \\
\hline abs coeff $/ \mathrm{mm}^{-1}$ & 7.994 & 0.37 \\
\hline$\theta$ range $/$ deg & 4.87 to 72.35 & 3.36 to 30.03 \\
\hline \multirow[t]{2}{*}{ reflns collected/indep reflns } & $24058 / 2370$ & $73335 / 11924$ \\
\hline & {$[R($ int $)=0.0418]$} & {$[R($ int $)=0.0255]$} \\
\hline max. and min transmn & 0.380 and 0.109 & 1.000 and 0.949 \\
\hline final $R 1$ indices & 0.0234 & 0.0296 \\
\hline$w R 2$ indices (all data) & 0.0243 & 0.0839 \\
\hline largest diff peak and hole/e $\AA^{3}$ & 0.61 and -0.46 & 0.51 and -0.24 \\
\hline
\end{tabular}

131.24, $132.62\left(C_{6} \mathrm{H}_{5}\right), 177.11\left(\mathrm{C}_{6} \mathrm{H}_{5} C\right) \mathrm{ppm} .{ }^{19} \mathrm{~F} \mathrm{NMR}(188 \mathrm{MHz}$, $\left.\mathrm{C}_{6} \mathrm{D}_{6}, 298 \mathrm{~K}\right): \delta-132.38\left(J_{\mathrm{Si}-\mathrm{F}}=219.3 \mathrm{~Hz}\right)$ ppm. ${ }^{29} \mathrm{Si} \mathrm{NMR}$ $\left(99 \mathrm{MHz}, \mathrm{C}_{6} \mathrm{D}_{6}, 298 \mathrm{~K}\right): \delta-124.91$ (quartet, $J_{\mathrm{Si}-\mathrm{F}}=219.3 \mathrm{~Hz}$ ) ppm.

$\mathbf{L}^{2} \mathbf{S i F}_{3}$ (2b). Tetrahydrofuran (THF, $50 \mathrm{~mL}$ ) was added to a mixture of $\mathbf{1 b}(1.57 \mathrm{~g}, 5.76 \mathrm{mmol})$ and $\mathrm{Me}_{3} \mathrm{SnF}$ at room temperature. After $5 \mathrm{~min}$ the $\mathrm{Me}_{3} \mathrm{SnF}$ had dissolved, resulting in a colorless solution. The volatiles were removed under vacuum to obtain a white solid. Recrystallization from toluene at $-35^{\circ} \mathrm{C}$ afforded colorless crystals of $\mathbf{2 b}(1.01 \mathrm{~g}, 79 \%)$. Anal. Calcd (\%) for $\mathrm{C}_{7} \mathrm{H}_{12} \mathrm{~F}_{3} \mathrm{~N}_{3} \mathrm{Si}(\mathrm{M}=223.27)$ : $\mathrm{C}, 37.66 ; \mathrm{H}, 5.42 ; \mathrm{N}, 18.82$. Found (\%): C, 37.59; H, 5.33; N, 18.64. ${ }^{1} \mathrm{H}$ NMR $(200 \mathrm{MHz}$, $\left.\mathrm{C}_{6} \mathrm{D}_{6}, 298 \mathrm{~K}\right): \delta 1.26\left(\mathrm{~m}, 4 \mathrm{H}, \mathrm{CH}_{2}\right), 2.31(\mathrm{t}, J=6.0 \mathrm{~Hz}, 4 \mathrm{H}$, $\left.\mathrm{CH}_{2}\right), 2.83\left(\mathrm{t}, \mathrm{J}=6.0 \mathrm{~Hz}, 4 \mathrm{H}, \mathrm{CH}_{2}\right) \mathrm{ppm} .{ }^{13} \mathrm{C}\left\{{ }^{1} \mathrm{H}\right\} \mathrm{NMR}$ $\left(125 \mathrm{MHz}, \mathrm{C}_{6} \mathrm{D}_{6}, 298 \mathrm{~K}\right): \delta 23.23\left(\mathrm{CH}_{2}\right), 39.56\left(\mathrm{CH}_{2}\right), 43.34$ $\left(\mathrm{CH}_{2}\right), 155.32\left(\mathrm{CN}_{3}\right)$ ppm. ${ }^{19} \mathrm{~F}$ NMR $\left(188 \mathrm{MHz}, \mathrm{C}_{6} \mathrm{D}_{6}, 298 \mathrm{~K}\right)$ : $\delta-137.76\left(J_{\mathrm{Si}-\mathrm{F}}=203.7 \mathrm{~Hz}\right) \mathrm{ppm} .{ }^{29} \mathrm{Si} \operatorname{NMR}\left(59 \mathrm{MHz}, \mathrm{C}_{6} \mathrm{D}_{6}\right.$, $298 \mathrm{~K}$ ): $\delta-127.96$ (quartet, $J_{\mathrm{Si}-\mathrm{F}}=203.7 \mathrm{~Hz}$ ) ppm.

$\mathbf{L}^{3} \mathbf{S i F}_{\mathbf{2}}(\mathbf{2 c})$. Compound $\mathbf{2 c}$ was prepared analogously to $\mathbf{2} \mathbf{b}$, using $1 \mathrm{c}(1.54 \mathrm{~g}, 3.72 \mathrm{mmol})$ and $\mathrm{Me}_{3} \mathrm{SnF}(1.37 \mathrm{~g}, 7.49 \mathrm{mmol})$, as colorless crystals $\left(1.11 \mathrm{~g}, 78 \%\right.$ ). Anal. Calcd. for $\mathrm{C}_{18} \mathrm{H}_{29} \mathrm{~F}_{2} \mathrm{~N}_{5} \mathrm{Si}$ $(\mathrm{M}=381.54): \mathrm{C}, 56.66 ; \mathrm{H}, 7.66 ; \mathrm{N}, 18.36$. Found (\%): C, 56.67; $\mathrm{H}, 7.55 ; \mathrm{N}, 18.23 .{ }^{1} \mathrm{H}$ NMR $\left(200 \mathrm{MHz}, \mathrm{C}_{6} \mathrm{D}_{6}, 298 \mathrm{~K}\right): \delta 1.14$ $\left(\mathrm{d}, J=6.7 \mathrm{~Hz}, 6 \mathrm{H}, \mathrm{CHMe} e_{2}\right), 1.23\left(\mathrm{~d}, J=6.6 \mathrm{~Hz}, 6 \mathrm{H}, \mathrm{CH} M e_{2}\right)$, $2.40\left(\mathrm{~s}, 6 \mathrm{H}, \mathrm{NM} e_{2}\right), 2.62$ (s, 3H, NMe 2 ), 2.81 (s, 3H, NMe $), 3.43$ (m, 2H, CHMe $), 7.00-7.09\left(\mathrm{~m}, 3 \mathrm{H}, \mathrm{C}_{6} \mathrm{H}_{3}\right)$ ppm. ${ }^{13} \mathrm{C}\left\{{ }^{1} \mathrm{H}\right\}$ NMR (125 MHz, $\left.\mathrm{C}_{6} \mathrm{D}_{6}, 298 \mathrm{~K}\right): \delta 24.31(\mathrm{CHMe}), 25.56$ $(\mathrm{CHMe}), 29.32\left(\mathrm{CHMe}_{2}\right), 37.23,37.66,40.31\left(\mathrm{~N} M e_{2}\right), 126.12$, $128.00,137.55\left(C_{6} \mathrm{H}_{3}\right), 147.07$ (ipso- $\left.C_{6} \mathrm{H}_{3}\right), 162.46,162.81$ $(\mathrm{NCN}) \mathrm{ppm} .{ }^{19} \mathrm{~F}$ NMR $\left(188 \mathrm{MHz}, \mathrm{C}_{6} \mathrm{D}_{6}, 298 \mathrm{~K}\right): \delta-135.54$ $\left(J_{\mathrm{Si}-\mathrm{F}}=206.6 \mathrm{~Hz}\right) \mathrm{ppm} .{ }^{29} \mathrm{Si} \mathrm{NMR}\left(99 \mathrm{MHz}, \mathrm{C}_{6} \mathrm{D}_{6}, 298 \mathrm{~K}\right)$ : $\delta-132.03\left(\mathrm{t}, J_{\mathrm{Si}-\mathrm{F}}=206.6 \mathrm{~Hz}\right) \mathrm{ppm}$.

Single Crystal X-ray Structure Determinations. Crystal data are summarized in Tables 1 and 2. Data for $\mathbf{1 c}$ and $\mathbf{2 a}$ were recorded on an Oxford Diffraction Xcalibur diffractometer using monochromated Mo K $\alpha$ radiation. Empirical multiscan absorption corrections were performed. Structural parameters were refined anisotropically on $F^{2}$ using the program SHELXL-97. ${ }^{12}$ Hydrogen atoms were included using a riding model or rigid methyl groups. A crystal of each of the compounds $\mathbf{1 b}$ and 2c was mounted on a Bruker three circle diffractometer

(12) Sheldrick, G. M. Acta Crystallogr. 2008, A 64, 112-122.
Table 2. Crystallographic Data and Structure Refinement for $2 \mathrm{a}$ and $\mathbf{2 c}$

\begin{tabular}{|c|c|c|}
\hline & $2 a$ & $(2 \mathrm{c})_{2}$ \\
\hline formula & $\mathrm{C}_{15} \mathrm{H}_{23} \mathrm{~F}_{3} \mathrm{~N}_{2} \mathrm{Si}$ & $\mathrm{C}_{36} \mathrm{H}_{58} \mathrm{~F}_{4} \mathrm{~N}_{10} \mathrm{Si}_{2}$ \\
\hline Fw & 316.44 & 763.08 \\
\hline cryst size $/ \mathrm{mm}$ & $0.28 \times 0.20 \times 0.12$ & $0.50 \times 0.25 \times 0.25$ \\
\hline cryst syst & triclinic & monoclinic \\
\hline space & $P \overline{1}$ & $C 2$ \\
\hline$T /{ }^{\circ} \mathrm{C}$ & -173 & -173 \\
\hline$a / \AA$ & $8.7620(9)$ & $14.4012(3)$ \\
\hline$b / \AA$ & $8.9751(9)$ & $14.4485(3)$ \\
\hline$c / \AA$ & 10. & $9.8332(2)$ \\
\hline$\alpha / \operatorname{deg}$ & 93.738 & 90 \\
\hline$\beta / \mathrm{deg}$ & 100.7 & $101.970(1)$ \\
\hline$\gamma / \mathrm{deg}$ & 107.182(9) & 90 \\
\hline$V / \AA^{3}$ & 804 & $2001.56(7)$ \\
\hline$D_{\text {calcd }} / \mathrm{g} \mathrm{cm}^{-3}$ & 1.307 & 1.270 \\
\hline$Z$ & 2 & 2 \\
\hline wave & 0.71 & 1.54184 \\
\hline & 0.17 & 1.29 \\
\hline$\theta$ range $/ \mathrm{deg}$ & 2.79 to 30.99 & 4.38 to 72.93 \\
\hline \multirow[t]{2}{*}{ reflns collected/indep reflns } & $23331 / 5091$ & $21748 / 3915$ \\
\hline & {$[R($ int $)=0.0308]$} & {$[R(\mathrm{int})=0.0276]$} \\
\hline $\max$. and $\mathrm{n}$ & 0.999 and 0.946 & 0.4701 and 0.3717 \\
\hline final $R 1$ indice & 0.0311 & 0.0242 \\
\hline$w R 2$ indic & 0.0876 & 0.0246 \\
\hline largest diff peak and hole/e $\AA^{3}$ & 0.38 and -0.20 & 0.21 and -0.15 \\
\hline
\end{tabular}

equipped with a SMART $6000 \mathrm{CCD}$ detector and a $\mathrm{Cu} \mathrm{K} \alpha$ rotating anode. Integration was performed with SAINT. ${ }^{13}$ For absorption and scaling of intensity data the SADABS program ${ }^{14}$ was used. Structures were solved using direct methods and refined by full-matrix least-squares against $F^{2}$ with SHELXL-97. ${ }^{12}$ Non-hydrogen atoms were refined anisotropically. For hydrogen, positions and isotropic displacement parameters were refined. The asymmetric unit of $\mathbf{2} \mathbf{c}$ contains half a molecule. For compound 1b, the Independent Atom Model (IAM) refinement starting values were used for Invariom refinement ${ }^{15}$ which is based on the Hansen and Coppens multipole formalism. ${ }^{16}$ This non-spherical atom refinement, which included reflections with $[F>3 \sigma(F)]$, was performed with XDLSM as part of the XD package. ${ }^{17} \mathrm{XD}$ input files were processed with the program Invariom Tool. ${ }^{18}$ In the Invariom refinement multipole parameters are fixed at theoretically predicted values, and only the positional and displacement parameters are refined, providing a more accurate structural model. Full details of the general Invariom modeling procedure can be found in literature. ${ }^{18}$

\section{Results and Discussion}

Synthesis and Characterization. The reaction of $\mathrm{L}^{1} \mathrm{SiCl}_{3}$ (1a) with $\mathrm{Me}_{3} \mathrm{SnF}$ in THF at room temperature led to the immediate dissolution of $\mathrm{Me}_{3} \mathrm{SnF}$ with the formation of $\mathrm{L}^{1} \mathrm{SiF}_{3}(\mathbf{2 a})$ with pentacoordinate silicon atom. (Scheme 1).

Deprotonation of $1,3,4,6,7,8$-hexahydro- $2 \mathrm{H}$-pyrimido[1,2-a]pyrimidine $\left(\mathrm{L}^{2} \mathrm{H}\right)$ with ${ }^{t} \mathrm{BuLi}$ and subsequent treatment with $\mathrm{SiCl}_{4}$ gave compound $\mathbf{1 b}$, which reacts with $\mathrm{Me}_{3} \mathrm{SnF}$ in $\mathrm{THF}$ to afford $\mathbf{2 b}$ (Scheme 2).

Reaction of $\mathrm{L}^{3^{\prime}} \mathrm{Li}$ (where $\mathrm{L}^{3^{\prime}}=\mathrm{Me}_{3} \mathrm{SiNC}\left(\mathrm{NMe}_{2}\right) \mathrm{NC}$ $\left(\mathrm{NMe}_{2}\right) \mathrm{NAr}$ and $\left.\mathrm{Ar}=2,6-{ }^{i} \mathrm{Pr}_{2} \mathrm{C}_{6} \mathrm{H}_{3}\right)$ with $\mathrm{SiCl}_{4}$ yielded

(13) APEX2, SAINT, and SHELXTL; Bruker AXS Inc.: Madison, WI, 2009

(14) Sheldrick, G. M. SADABS; University of Göttingen: Göttingen, Germany, 2009.

(15) Dittrich, B.; Hübschle, C. B.; Messerschmidt, M.; Kalinowski, R.; Girnt, D.; Luger, P. Acta Crystallogr. 2005, A 61, 314-320.

(16) Hansen, N. K.; Coppens, P. Acta Crystallogr. 1978, A 34, 909-921.

(17) Koritsánszky, T.; Richter, T.; Macchi, P.; Volkov, A.; Gatti, C.; Howard, S.; Mallinson, P. R.; Farrugia, L.; Su, Z. W.; Hansen, N. K. XD, XDLSM; Freie Universität Berlin: Berlin, Germany, 2003.

(18) Hübschle, C. B.; Luger, P.; Dittrich, B. J. Appl. Crystallogr. 2007, 40, 623-627. 
compound 1c with the elimination of $\mathrm{LiCl}$ and $\mathrm{Me}_{3} \mathrm{SiCl}$. In contrast to transition metal ${ }^{11}$ complexes, where the triazapentadienate ligand $\left(\mathrm{L}^{3^{\prime}}\right)^{-}$is monoanionic, $\mathrm{L}^{3}$ in 1c is dianionic $\left(\mathrm{L}^{3}\right)^{2-}$ because of the elimination of $\mathrm{Me}_{3} \mathrm{SiCl}$ group. Treatment of $\mathbf{1 c}$ with $\mathrm{Me}_{3} \mathrm{SnF}$ affords compound 2c (Scheme 3).

Base-Induced Disproportionation of $\mathrm{Si}_{2} \mathrm{Cl}_{6}$ to Stable Silylenes. Reaction of $\mathrm{Si}_{2} \mathrm{Cl}_{6}$ with lithium amidinate $\left(\mathrm{L}^{1} \mathrm{Li}\right)$ resulted in the formation of trichlorosilane (1a) and monochlorosilylene $\left(\mathrm{L}^{1} \mathrm{SiCl}\right)(3)^{6 \mathrm{a}}$ (Scheme 4) instead of the expected diamidinatotetrachlorodisilane $\left(\mathrm{L}^{1} \mathrm{SiCl}_{2} \mathrm{SiCl}_{2} \mathrm{~L}^{1}\right)$. This finding offers a new one pot method for the preparation of base-stabilized silylenes. Similarly, reaction of $\mathrm{Si}_{2} \mathrm{Cl}_{6}$ with 2 equiv of $\mathrm{NHC}$ afforded $\mathrm{NHC} \cdot \mathrm{SiCl}_{2}(\mathrm{NHC}$ is $\mathrm{IPr}=1$,

\section{Scheme 1}<smiles></smiles>

1a
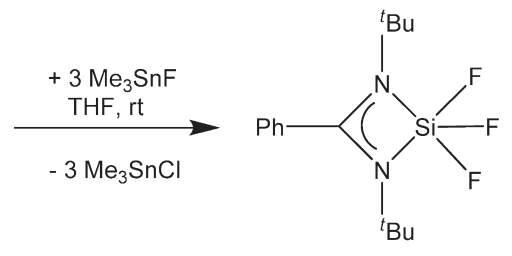

2a

\section{Scheme 2}
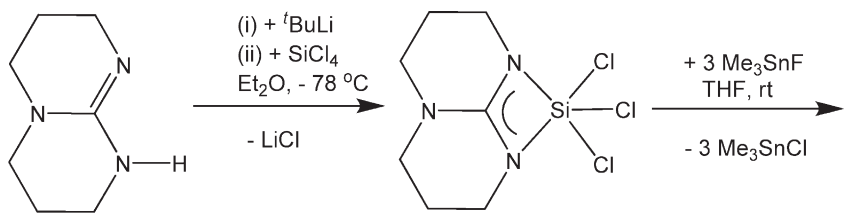

1b

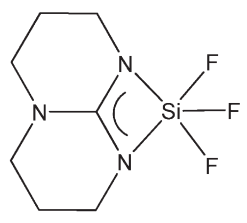

$2 b$ 3-bis(2,6-diisopropylphenyl)imidazol-2-ylidene 4a or IMes =1,3-bis(2,4,6-trimethylphenyl)imidazol-2-ylidene 4b $)^{6 \mathrm{~d}}$ and $\mathrm{NHC} \cdot \mathrm{SiCl}_{4}(\mathrm{NHC}=\mathrm{IPr} \mathbf{5 a} \text { or IMes } \mathbf{5 b})^{6 \mathrm{c}, \mathrm{d}}$ instead of $\left(\mathrm{NHC} \cdot \mathrm{SiCl}_{3}\right)_{2}$. The physical properties and NMR spectral data of these compounds $\mathbf{1 a}, \mathbf{3}, \mathbf{4 a}, \mathbf{4 b}, \mathbf{5 a}, \mathbf{5 b}$ are identical with those reported in literature. ${ }^{\text {a,c, d }}$ Thermal $^{19}$ and base-induced ${ }^{20}$ disproportionation of $\mathrm{Si}_{2} \mathrm{Cl}_{6}$ to $\mathrm{SiCl}_{2}$ and $\mathrm{SiCl}_{4}$ is known, and trapping experiments of $\mathrm{SiCl}_{2}$ with unsaturated substrates have been reported in the literature, ${ }^{21}$ but no stable silylenes have been isolated using this method. Therefore, careful selection of the ligands and their treatment with $\mathrm{Si}_{2} \mathrm{Cl}_{6}$ may serve as an indicator of silylene stability for a given ligand. Further investigations in this direction are continuing, and the results will be published in due course.

Compounds $\mathbf{1 b}, \mathbf{1 c}, \mathbf{2 a}, \mathbf{2 b}$, and $\mathbf{2 c}$ are colorless crystalline solids, soluble in common organic solvents, and are stable under an inert atmosphere. These compounds were characterized by elemental analyses, ${ }^{1} \mathrm{H},{ }^{13} \mathrm{C},{ }^{19} \mathrm{~F}$, and ${ }^{29} \mathrm{Si}$ NMR spectroscopic studies. Compound 2a shows ${ }^{1} \mathrm{H}$ and ${ }^{13} \mathrm{C}$ NMR resonances for the amidinato moiety. ${ }^{1} \mathrm{H}$ and ${ }^{13} \mathrm{C}$ NMR spectra of $\mathbf{1 b}$ and $\mathbf{2} \mathbf{b}$ exhibit resonances for the cyclic guanidinate ligand coordinated to the silicon atom in a bidentate fashion. The conversion of monofunctional triazapentadienate to bifunctional ligand in the formation of compound $\mathbf{1 c}$ can be clearly seen in the ${ }^{1} \mathrm{H}$ and ${ }^{13} \mathrm{C}$ NMR spectra. The resonance from the $\mathrm{Me}_{3} \mathrm{Si}$ group is absent, and the methyl groups of the $\mathrm{NMe}_{2}$ substituents appear as three sets of resonances in the ${ }^{1} \mathrm{H}$ and ${ }^{13} \mathrm{C}$ NMR spectra. In the ${ }^{1} \mathrm{H}$ NMR spectrum of $\mathbf{1 c}$, one resonance at $\delta 2.40\left(\mathrm{~s}, 6 \mathrm{H}, \mathrm{Me}_{2} \mathrm{NC}=\mathrm{N}\right) \mathrm{ppm}$ is preliminarily assigned to the $\mathrm{NMe}_{2}$ group attached to the carbon atom of the $C=\mathrm{N}-\mathrm{Si}$ moiety, whereas the two resonances at $\delta 2.62$ $\left(\mathrm{s}, 3 \mathrm{H}, \mathrm{Me}_{2} \mathrm{~N}\right)$ and $2.81\left(\mathrm{~s}, 3 \mathrm{H}, \mathrm{Me}_{2} \mathrm{~N}\right) \mathrm{ppm}$ are assigned to the $\mathrm{NMe}_{2}$ group at the carbon atom of the $C-\mathrm{N}-\mathrm{Si}$ moiety. The presence of a bulky $2,6-{ }^{i} \mathrm{Pr}_{2} \mathrm{C}_{6} \mathrm{H}_{3}$ group at the adjacent amine nitrogen restricts the free rotation of the $\mathrm{NMe}_{2}$ group with the consequence that at room temperature two singlets appear. Compounds $\mathbf{2 a - 2 c}$ show the expected resonances in the ${ }^{1} \mathrm{H}$ and ${ }^{13} \mathrm{C}$ NMR spectra. 2a, $\mathbf{2 b}$, and $2 \mathbf{c}$ exhibit ${ }^{19} \mathrm{~F}$ NMR resonances at $\delta-132.38$,

Scheme 3
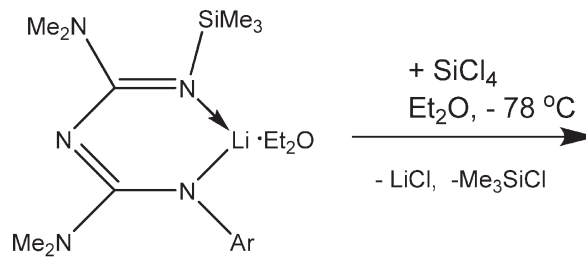

$\mathrm{L}^{3} \mathrm{Li}$

$\operatorname{Ar}=2,6$-diisopropylphenyl

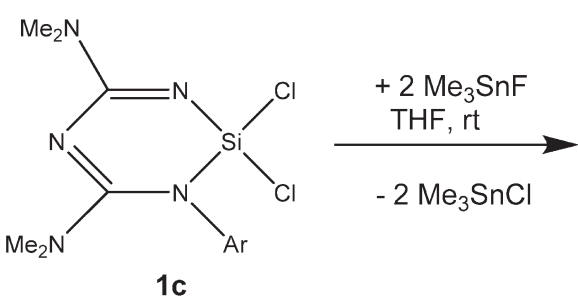

1c

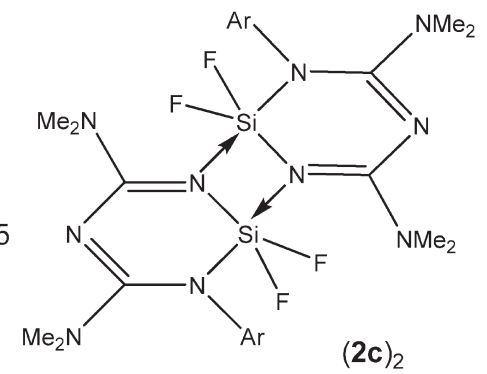




\section{Scheme 4}

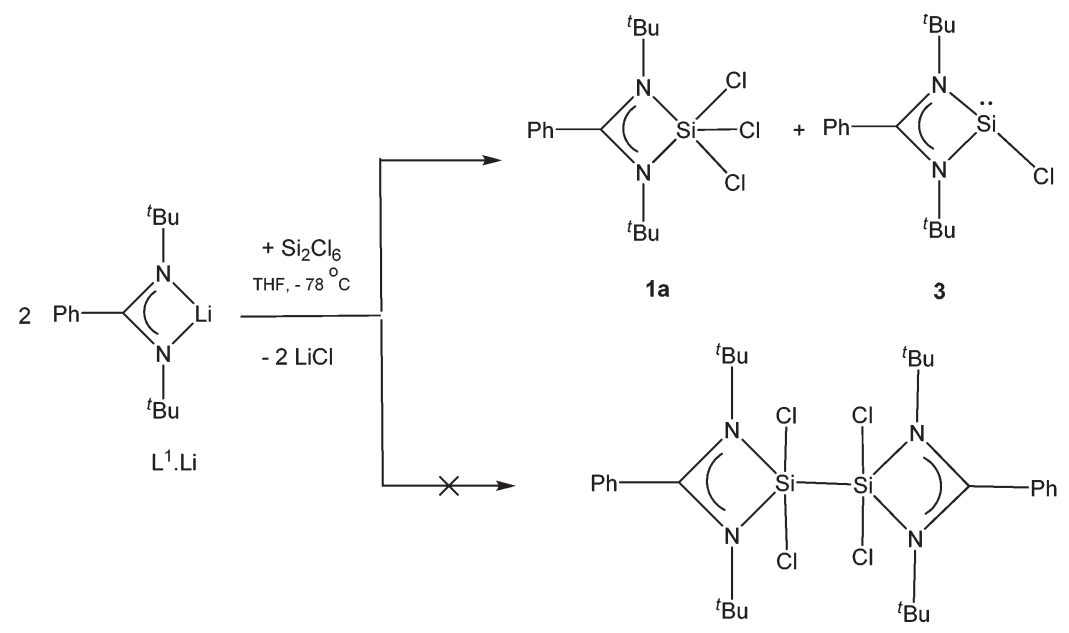

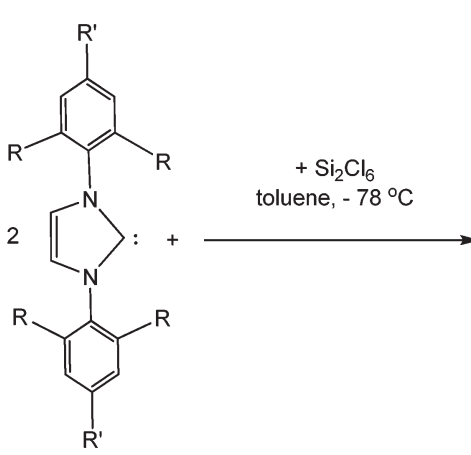

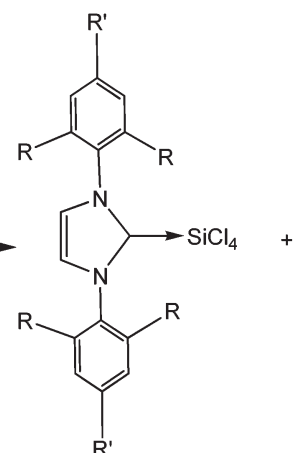

4a: $\mathrm{R}={ }^{i} \mathrm{Pr} ; \mathrm{R}^{\prime}=\mathrm{H}$

$\mathrm{IPr}: \mathrm{R}={ }^{i} \mathrm{Pr} ; \mathrm{R}^{\prime}=\mathrm{H}$ IMes: $R=R^{\prime}=M e$<smiles>[R]c1cc([R])c(N2C=CN(c3c([R])cc([R])cc3[R])C2[C@H]2C[C@H](C)C2[R])c([R])c1</smiles>

5a: $:{ }^{i} \mathrm{Pr} ; \mathrm{R}^{\prime}=\mathrm{H}$

$\mathbf{5 b}: \mathbf{R}=\mathrm{R}^{\prime}=\mathrm{Me}$
$-137.76,-135.54 \mathrm{ppm}$, respectively, with $J_{\mathrm{Si}-\mathrm{F}}$ ranging from 203 to $219 \mathrm{~Hz}$. Compounds $\mathbf{1 b}, \mathbf{2 a}$, and $\mathbf{2 b}$ each show a sharp ${ }^{29} \mathrm{Si}$ NMR resonance in the range $\delta-103.42$ to $-127.96 \mathrm{ppm}$, consistent for pentacoordinate ${ }^{6}$ silicon. The presence of tetracoordinate silicon in $\mathbf{1 c}$, in which triazapentadienate is a bifunctional ligand, is further supported by a sharp ${ }^{29} \mathrm{Si}$ NMR resonance at $\delta-30.57 \mathrm{ppm}$. The appearance of the ${ }^{29} \mathrm{Si}$ NMR resonance at $\delta-132.03$ $\mathrm{ppm}$ for $\mathbf{2 c}$ indicates the retention of pentacoordinate silicon in solution. Single crystal X-ray structural analysis shows the dimeric nature of $\mathbf{2} \mathbf{c}$ with pentacoordinate silicon.

Single Crystal X-ray Structures. Molecular structures of compounds $\mathbf{1 b}, \mathbf{1 c}, \mathbf{2} \mathbf{a}$, and $\mathbf{2 c}$ were determined by single crystal X-ray crystallography and are shown in Figures 1-4. Crystallographic data for $\mathbf{1 b}, \mathbf{1 c}, \mathbf{2 a}$, and $\mathbf{2 c}$

(19) (a) Goldberg, N.; Ogden, J. S.; Almond, M. J.; Walsh, R.; Cannady, J. P.; Becerra, R.; Lee, J. A. Phys. Chem. Chem. Phys. 2003, 5, 5371-5377. (b) Heinicke, J.; Vorwerk, D.; Zimmermann, V. J. Anal. Appl. Pyrolysis 1994, 28 , 93-105.

(20) (a) Kummer, D.; Köster, H.; Speck, M. Angew. Chem., Int. Ed. 1969, 8, 599-600. Kummer, D.; Köster, H.; Speck, M. Angew. Chem. 1969, 81, 574575. (b) Kummer, D.; Köster, H. Angew. Chem., Int. Ed. 1969, 8, 878-879. Kummer, D.; Köster, H. Angew. Chem. 1969, 81, 897-898.

(21) (a) du Mont, W.-W.; Gust, T.; Seppälä, E.; Wismach, C.; Jones, P. G.; Ernst, L.; Grunenberg, J.; Marsmann, H. C. Angew. Chem., Int. Ed. 2002, 41, 3829-3832. Angew. Chem. 2002, 114, 3977-3979. (b) Mahnke, J.; Zanin, A.; du Mont, W.-W.; Ruthe, F.; Jones, P. G. Z. Anorg. Allg. Chem. 1998, $624,1447-1454$ are summarized in Tables 1 and 2. The molecular structure of complex $\mathbf{1 b}$, which crystallizes in the monoclinic space group $C 2 / c$, is shown in Figure 1. The silicon atom in $\mathbf{1 b}$ is pentacoordinate, and the bond angles around silicon indicate a distorted trigonal-bipyramidal geometry, whereby $\mathrm{N}(2)$ and $\mathrm{Cl}(1)$ occupy axial and $\mathrm{N}(1), \mathrm{Cl}(2), \mathrm{Cl}(3)$ equatorial positions. The main distortion is imposed within the four-membered ring by the small bite angle $\left(71.60(4)^{\circ}\right)$ of the bicyclic guanidinate $\left(\mathrm{L}^{2}\right)$ ligand. ${ }^{22}$ The $\mathrm{C}(1)-\mathrm{N}(1)(1.3570(12)(\AA))$ and $\mathrm{C}(1)-\mathrm{N}(2)(1.3280(14)(\AA))$ bond distances are indicative of $\pi$-delocalization from the amide nitrogen. As expected, the equatorial $\mathrm{Si}(1)-\mathrm{Cl}(2)$ and $\mathrm{Si}(1)-\mathrm{Cl}(3)$ bonds are noticeably shorter than the axial $\mathrm{Si}(1)-\mathrm{Cl}(1)$ bond. A similar trend can be seen in the $\mathrm{Si}-\mathrm{N}$ bond distances $\mathrm{Si}(1)-\mathrm{N}(1)$ 1.7831(10) and $\mathrm{Si}(1)-\mathrm{N}(2) 1.8684(8) \AA$.

Complex 1c crystallizes in the triclinic space group $P \overline{1}$ with two independent molecules, which are however closely related (rms deviation $0.20 \AA$ ). It features a distorted

(22) (a) Kummer, D.; Halim, S. H. A.; Kuhs, W.; Mattern, G. J. Organomet. Chem. 1993, 446, 51-65. (b) Coles, M. P.; Hitchock, P. B. Dalton Trans. 2001, 1169-1171. (c) Coles, M. P.; Hitchcock, P. B. Organometallics 2003, 22, 5201-5211. (d) Oakley, S. H.; Coles, M. P.; Hitchcock, P. B. Dalton Trans. 2004, 1113-1114. (e) Oakley, S. H.; Coles, M. P.; Hitchcock, P. B. Inorg. Chem. 2004, 43, 5168-5172. (f) Soria, D. B.; Grundy, J.; Coles, M. P.; Hitchcock, P. B. J. Organomet. Chem. 2005, 690, 2278-2284. (g) Maaranen, J.; Andell, O. S.; Vanne, T; Mutikainen, I. J. Organomet. Chem. 2006, 691, 240-246. (h) Coles, M. P.; Sözerli, S. E.; Smith, J. D.; Hitchcock, P. B. Organometallics 2007, 26, 6691-6693. 


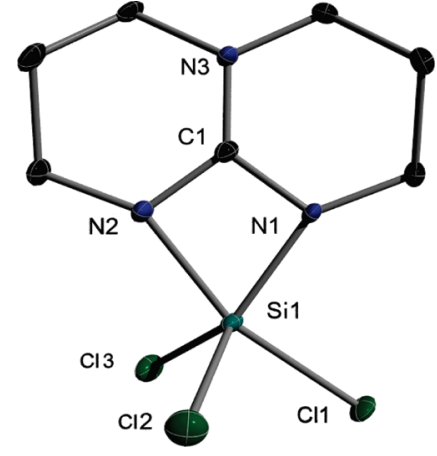

Figure 1. Molecular structure of $\mathbf{1 b}$. Anisotropic displacement parameters are depicted at the $50 \%$ probability level. $\mathrm{H}$ atoms are omitted for clarity. Selected bond distances $(\AA)$ and angles (deg): $\operatorname{Si}(1)-\mathrm{N}(1)$ 1.7831(10), $\mathrm{Si}(1)-\mathrm{N}(2)$ 1.8684(8), $\mathrm{Si}(1)-\mathrm{Cl}(1) 2.1669(3), \mathrm{Si}(1)-\mathrm{Cl}(2)$ 2.0778(3), $\mathrm{Si}(1)-\mathrm{Cl}(3) 2.0900(3), \mathrm{N}(2)-\mathrm{C}(1)$ 1.3280(14), $\mathrm{N}(1)-\mathrm{C}(1)$ $1.3570(12), \mathrm{N}(3)-\mathrm{C}(1) 1.3144(13) ; \mathrm{N}(1)-\mathrm{Si}(1)-\mathrm{N}(2) 71.60(4), \mathrm{N}(1)-$ $\mathrm{Si}(1)-\mathrm{Cl}(2)$ 117.37(3), N(2)-Si(1)-Cl(2) 97.73(3), N(1)-Si(1)-Cl(3) 133.49(3), $\mathrm{N}(2)-\mathrm{Si}(1)-\mathrm{Cl}(3)$ 93.18(3), $\mathrm{Cl}(2)-\mathrm{Si}(1)-\mathrm{Cl}(3)$ 107.924(15), $\mathrm{N}(1)-\mathrm{Si}(1)-\mathrm{Cl}(1)$ 91.56(3), N(2)-Si(1)-Cl(1) 161.67(3), $\mathrm{Cl}(2)-\mathrm{Si}(1)-$ $\mathrm{Cl}(1)$ 96.472(12).

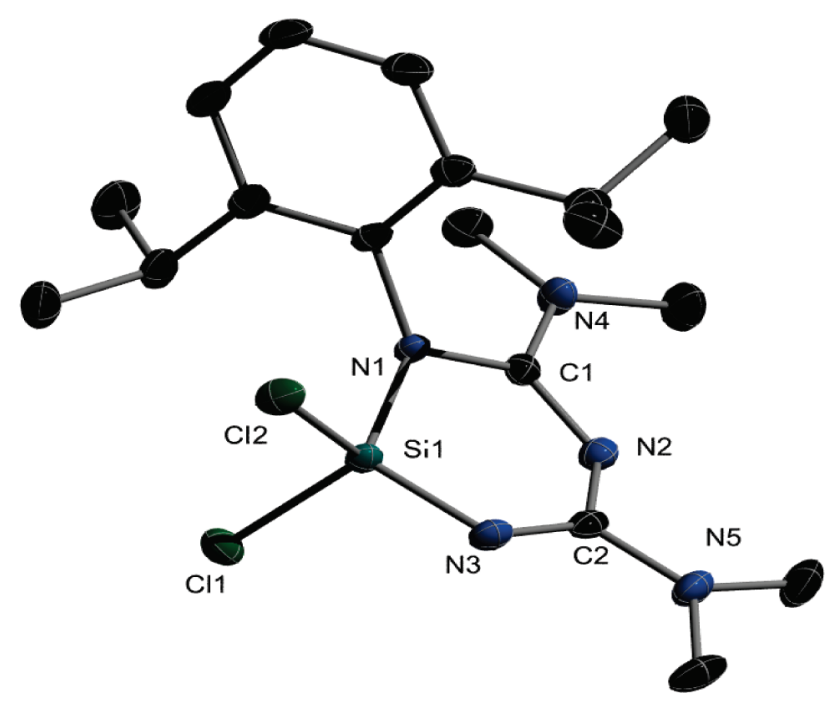

Figure 2. Molecular structure of 1c. Anisotropic displacement parameters are depicted at the $50 \%$ probability level. $\mathrm{H}$ atoms are omitted for clarity. Selected bond distances $(\AA)$ and angles (deg) (second values refer to the second independent molecule): $\mathrm{Si}(1)-\mathrm{N}(3)$ 1.6501(10), 1.6479(10), $\mathrm{Si}(1)-\mathrm{N}(1)$ 1.7494(9), 1.7487(9), $\mathrm{Si}(1)-\mathrm{Cl}(1)$ 2.0568(4), 2.0533(4), $\mathrm{Si}(1)-\mathrm{Cl}(2) 2.0361(4), 2.0362(4), \mathrm{N}(1)-\mathrm{C}(1) 1.4023$ (14), $1.4019(14), \mathrm{N}(2)-\mathrm{C}(1) 1.3113(14), 1.3120(14), \mathrm{N}(2)-\mathrm{C}(2) 1.3824$ (15),

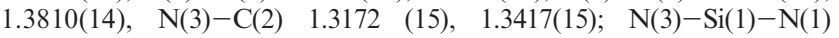
$108.12(5), 108.70(5), \mathrm{N}(3)-\mathrm{Si}(1)-\mathrm{Cl}(2) \quad 113.72(4), 113.31(4), \mathrm{N}(1)-$ $\mathrm{Si}(1)-\mathrm{Cl}(2)$ 108.31(3), 108.46(3), N(3)-Si(1)-Cl(1) 111.12(4), 110.05(4), $\mathrm{N}(1)-\mathrm{Si}(1)-\mathrm{Cl}(1) 110.56(3), 112.07(3), \mathrm{Cl}(2)-\mathrm{Si}(1)-\mathrm{Cl}(1)$ 104.962(18), 104.254(19).

tetrahedral geometry at the tetracoordinate silicon (Figure 2). The NCNCN moiety shows, beginning at N(3), a shortlong-short-long pattern of $\mathrm{C}-\mathrm{N}$ bond distances. The $\mathrm{Si}(1)-\mathrm{N}(3)$ bond $(1.6501(10), 1.6479(10) \AA$ in the two molecules) is significantly shorter than $\mathrm{Si}(1)-\mathrm{N}(1)$ (1.7494(9), $1.7487(9) \AA$ ), associated with the presence of the bulky 2,6- ${ }^{i} \mathrm{Pr}_{2} \mathrm{C}_{6} \mathrm{H}_{3}$ group on $\mathrm{N}(1)$ compared to the unsubstituted N(3). The $\mathrm{Si}-\mathrm{Cl}$ bond distances (av $2.046 \AA$ ) are

(23) Ghadwal, R. S.; Roesky, H. W.; Merkel, S.; Stalke, D. Chem.-Eur. J. 2010, 16, 85-88.

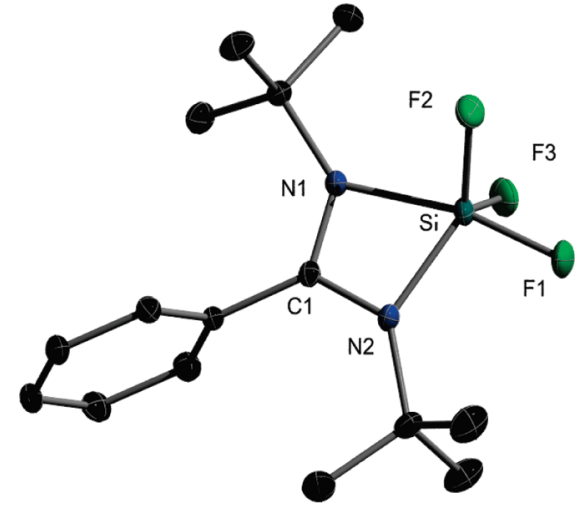

Figure 3. Molecular structure of 2a. Anisotropic displacement parameters are depicted at the $50 \%$ probability level. $\mathrm{H}$ atoms are omitted for clarity. Selected bond distances $(\AA)$ and angles (deg): $\mathrm{Si}(1)-\mathrm{F}(1) 1.6214(6)$, $\mathrm{Si}(1)-\mathrm{F}(2)$ 1.5988(6), Si(1)-F(3) 1.5947(7), Si(1) - N(1) 1.9120 (8), Si(1)$\mathrm{N}(2) 1.8026(8) ; \mathrm{F}(2)-\mathrm{Si}(1)-\mathrm{F}(1) 94.34(3), \mathrm{F}(3)-\mathrm{Si}(1)-\mathrm{N}(2) 120.70(4)$, $\mathrm{F}(3)-\mathrm{Si}(1)-\mathrm{N}(1)$ 92.57(3), $\mathrm{F}(1)-\mathrm{Si}(1)-\mathrm{N}(1)$ 167.31(3), N(2)-Si(1)$\mathrm{N}(1) 70.25(3)$

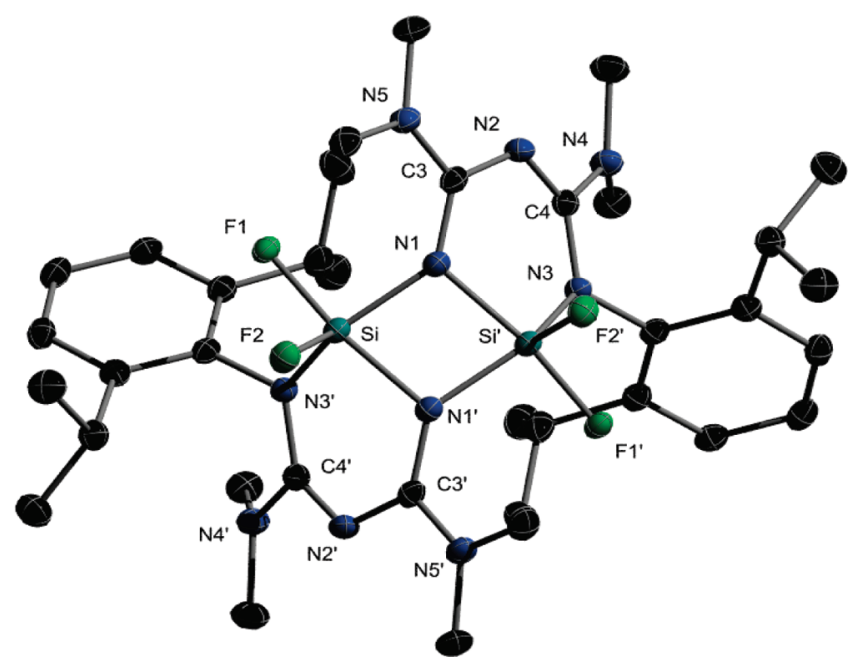

Figure 4. Molecular structure of $(\mathbf{2 c})_{2}$. Anisotropic displacement parameters are depicted at the $50 \%$ probability level. $\mathrm{H}$ atoms are omitted for clarity. Primes indicate symmetry-generated atoms. Selected bond distances $(\AA)$ and angles (deg): $\mathrm{Si}-\mathrm{F}(1)$ 1.6401(7), $\mathrm{Si}-\mathrm{F}(2)$ 1.6218(8), $\mathrm{Si}-$ $\mathrm{N}(1)$ 1.7685(10), Si-N(1) 1.8138(9); F(2)-Si-F(1) 93.27(4), F(2)$\mathrm{Si}-\mathrm{N}(1)$ 119.41(5), $\mathrm{F}(1)-\mathrm{Si}-\mathrm{N}(1)$ 93.83(4), F(1)-Si-N(1)' 170.20(4), $\mathrm{Si}-\mathrm{N}(1)-\mathrm{Si}^{\prime}$ 101.28(4), N(1)-Si'-N(3) 91.24(4).

consistent with known values of compounds with tetracoordinate silicon. ${ }^{23}$

Compound 2a crystallizes in the triclinic space group $P \overline{1}$ with distorted trigonal bipyramidal geometry around the pentacoordinate silicon atom (Figure 3). The atoms $\mathrm{N}(1)$ and $\mathrm{F}(1)$ occupy the axial positions, whereas $\mathrm{N}(2)$, $\mathrm{F}(2)$, and $\mathrm{F}(3)$ are equatorial. Analogously to compound $\mathbf{1 b}$, the differently disposed nitrogen atoms of the bidentate amidinate $\left(\mathrm{L}^{1}\right)$ ligand cause the main distortion, with a $\mathrm{N}(1)-\mathrm{Si}(1)-\mathrm{N}(2)$ bond angle of $70.25(3)^{\circ}$, and the axial $\mathrm{Si}(1)-\mathrm{F}(1)$ bond $(1.6214(6) \AA)$ is slightly longer than its equatorial counterparts (av 1.5967(7) $\AA$ ). ${ }^{2 \mathrm{~s}}$ Similarly, the $\mathrm{Si}-\mathrm{N}$ bond distances are 1.8026(8) and 1.9120(8) A respectively for the axial and equatorial $\mathrm{Si}-\mathrm{N}$ bonds. The $\mathrm{C}(1)-$ $\mathrm{N}(1)(1.3135(11) \AA$ and $\mathrm{C}(1)-\mathrm{N}(2)(1.3641(10) \AA)$ bond lengths are indicative of $\pi$-delocalization through the amide nitrogen. 
The molecular structure of compound $\mathbf{2} \mathbf{c}$ is shown in Figure 4. 2c crystallizes in the monoclinic space group $C 2$ as a dimer with imposed 2-fold symmetry. The dimeric nature of $\mathbf{2 c}$ also persists in solution at room temperature. The ${ }^{29} \mathrm{Si}$ NMR spectrum of $2 \mathrm{c}$ displays a triplet at $\delta-132.03 \mathrm{ppm}$ consistent with a pentacoordinate silicon atom. Clearly the monomeric molecules of $\mathrm{L}^{3} \mathrm{SiF}_{2}(\mathbf{2 c})$ react in a $[2+2]$ cycloaddition at the $\mathrm{Si}-\mathrm{N}$ bonds to yield dimeric $\left(\mathrm{L}^{3} \mathrm{SiF}_{2}\right)_{2}(\mathbf{2} \mathrm{c})_{2}$. Each of the silicon atoms features distorted trigonal-bipyramidal geometry. The nitrogen atoms of ligand $\mathrm{L}^{3}$ occupy one axial and one equatorial position at the same silicon atom. The second axial position is filled by one fluorine atom, and the remaining equatorial positions are occupied by the second fluorine atom and symmetry-generated nitrogen. The usual pattern of bond lengths is observed.

\section{Conclusion}

A direct fluorination method for the preparation of fluorides with pentacoordinate silicon based on amidinate, guanidinate, and triazapentadienate ligands is reported. A one pot method is discussed for the generation of stable silylenes from base-induced disproportionation of $\mathrm{Si}_{2} \mathrm{Cl}_{6}$. This method may serve as a key reaction for generating silylenes and trapping the silylene intermediates with various bases. Invariom refinement involving non-spherical scattering factors of the Hansen-Coppens multipole model was performed for $\mathbf{1 b}$.

Acknowledgment. Financial support from the Deutsche Forschungsgemeinschaft is gratefully acknowledged.

Supporting Information Available: Crystallographic data for complexes $\mathbf{1 b}, \mathbf{1 c}, \mathbf{2 a}$, and $\mathbf{2 c}$ as CIF files. This material is available free of charge via the Internet at http://pubs.acs.org. 